Wir arbeiten an unserem Gehirn mit unseren Gefühlen, Gedanken und Bewegungen. Dadurch wird das Ego entwickelt, das in dieser materiellen Welt mittels eines Gehirns möglich ist. Wir sind durch die Materie "Verzauberte», aber wir schöpfen unsere Realität aus Sir John Eccles (Nobelpreisträger) Quantensuppe, resp. aus dem universellen Vakuum. Inspiration und Intuition erleben wir, wenn das Geplapper der linken Hirnhälfte einmal aufhört, in einem Moment der Zeitlosigkeit, bei Meditation oder Kontemplation. Diese sind nie berechenbar. Bei einem Ausfall der linken Hirnhälfte durch eine Embolie, wie sie die Neuroanatomin Jill Bolte Taylor erlebte, ist das ekstatische Erleben der Einheit in der Zeitlosigkeit möglich. Und natürlich auch bei Nahtoderfahrungen, wie ich sie in meinem Buch
Hinter den Kulissen der Welt, ewige Ruhe? beschrieb. Die Zeit ist ja ein Kunstprodukt der 3D-Welt. Angelus Silesius definierte die Zeit sehr treffend: Du selber machst die Zeit, das Uhrwerk sind die Sinnen, hemmst du die Unruh nur, so ist die Zeit von hinnen.

Das grösste Problem sind unsere mentalen Bewertungen. Dazu gehören die Urteile und Definitionen. Definitionen verhindern das Erkennen undefinierter Wahrnehmungen. Die Urteile über uns und andere machen uns unglücklich. Mit dem Verstand allein bringen wir diese nicht weg, auch wenn wir sie als unrichtig erkannt haben. Dazu braucht es die Liebe, die Vergebung und das Mitgefühl, manchmal noch ein Geschenk an das verletzte Ego. Engel würden uns dabei sehr gerne helfen. Nach dem niederländischen Arzt Moolenburgh, der
1985 Befragungen über Engelerfahrungen bei seinen Patienten durchführte, ist die rechte Hirnhälfte auch die Empfangsstation für den «Engelkurzwellensender». Umgerechnet auf die Population der Niederlande damals käme er auf eine Zahl von stattlichen 150000 Menschen, die eine Engelerfahrung erlebten.

Das Grundproblem ist die gängige Vorstellung von Zeit und Materie. Beides ist eine Illusion, die wir miterschaffen und in der wir leben. Wir sind Schöpfer. Unser Gehirn produziert nicht das Bewusstsein. Unser Bewusstsein prägt und entwickelt das Gehirn.

Dr. med. Hedi Meierhans, Maseltrangen

1 Taverna E. Entzauberung. Schweiz Ärztzeitung. 2015;96(22):810-1.

\title{
Mitteilungen
}

\section{Facharztprüfung \\ Facharztprüfung zur Erlangung des Facharzttitels für Psychiatrie und Psychotherapie 2. Teil 2016}

Ort und Datum

Genf: Samstag, 11. Juni 2016

Lugano: Samstag, 11. Juni 2016

Bern: Samstag, 18. Juni 2016

Wil: Samstag, 18. Juni 2016

Olten: Samstag, 11. Juni 2016

\section{Anmeldefrist: 6. Januar 2016 um 16.00 Uhr}

Weitere Informationen finden Sie auf der Website des SIWF unter www.siwf.ch $\rightarrow$ Fachgebiete $\rightarrow$ Facharzttitel und Schwerpunkte (Weiterbildung) Psychiatrie und Psychotherapie.

\section{Facharztprüfung \\ Facharztprüfung zur Erlangung des Facharzttitels für Psychiatrie und Psychotherapie 1. Teil 2016 \\ Ort: Bern \\ Datum: Donnerstag, 25. August 2016 \\ Anmeldefrist: 10. Juni 2016 \\ Weitere Informationen finden Sie auf der Website des SIWF unter www.siwf.ch $\rightarrow$ Fachgebiete Facharzttitel und Schwer- punkte (Weiterbildung) $\rightarrow$ Psychiatrie und Psychotherapie.}

\section{Hilfskasse für Schweizer Ärzte Eingegangene Spenden}

Vom 1. April bis 30. Juni 2015 sind 20 Spenden im Gesamtbetrag von 23460.80 Franken eingegangen. Der Stiftungsrat der Hilfskasse für Schweizer Ärzte freut sich sehr, diese Gaben bekanntgeben zu dürfen, und dankt allen Spendern recht herzlich. Damit die Spenden in voller Höhe den Destinatären zukommen, haben wir uns entschlossen, für Spenden unter 500 Franken auf den Versand von persönlichen Dankschreiben zu verzichten. Wir hoffen sehr, dass diese Massnahme bei allen Spendern auf Verständnis stösst.

\section{Climate Change \\ Climate protection: Keep It In The Ground campaign}

Climate change has a profound impact on our wellbeing, today and in the future [1] . Therefore, The Guardian has started a new campaign. Leading health experts, including the editors of the British Medical Journal and the Lancet, are supporting the Keep It In the Ground campaign, which calls on the Wellcome Trust and Gates Foundation to divest from the 200 largest fossil fuel companies worldwide over the next five years. If you as a health professional also want to protect the environment, you are invited to sign the open letter published in The Guardian.

For further information, please have a look on the webpage www.theguardian.com $\rightarrow$ environment $\rightarrow$ climate change

1 Urbinello D, Künzli N. Wie der Klimawandel unsere Gesundheit beeinflusst. Schweiz Ärztezeitung 2015;96(31-32):1087-1088. 